\title{
CFD Simulations and External Shape Optimization of Missile with Wing and Tailfin Configuration to Improve Aerodynamic Performance
}

\author{
A. Şumnu ${ }^{1 \dagger}$ and İ. H. Güzelbey ${ }^{2}$ \\ ${ }^{I}$ Department of Aerospace Engineering, İskenderun Technical University, Hatay, 31200, Turkey \\ ${ }^{2}$ Department of Mechanical Engineering, Hasan Kalyoncu University, Ankara, 27620, Turkey \\ †Corresponding Author Email: ahmet.sumnu@iste.edu.tr
}

(Received February 4, 2021; accepted June 16, 2021)

\begin{abstract}
The wing of missile can be considered as an effective factor for determination of lift to drag ratio. However, there are few studies that investigate wing effect on missile aerodynamics. Therefore, the purpose of this study is to indicate wing effect on the missile aerodynamics and optimize wing geometry for enhancement of aerodynamic efficiency. The missile designed tail-fin configuration is selected from a previous study which contains experimental data. In the beginning of study, Computational Fluid Dynamics (CFD) simulations of selected missile are performed and compared with experimental data. Wing is then mounted to the selected missile and CFD solution is repeated for modified missile at $6^{\circ}$ angle of attach (AoA) and subsonic and supersonic speeds. The modified missile shows good performance in point of aerodynamics when compared with baseline missile model. In addition, wing geometry is optimized to improve aerodynamic performance using Multi-Objective Genetic Algorithm (MOGA). Objective functions are determined as lift and drag coefficients. Wing geometry parameters are determined as design variables for optimization. After the optimization process, the results are showed that the aerodynamic coefficients are improved when compared with baseline geometry. In addition, response surface analysis is presented to show which design parameters are more effective on drag and lift forces. The findings of study show that optimum results are more efficient in terms of performance. CFD solution method and the optimization procedure can be applied to design or optimize for different geometry.
\end{abstract}

Keywords: Missile aerodynamics; Genetic algorithm; Multi-objective optimization.

\section{NOMENCLATURE}

$\begin{array}{ll}C_{D} & \text { aerodynamic drag coefficient } \\ C_{L} & \text { aerodynamic lift coefficient } \\ \mathrm{E} & \text { total energy } \\ F_{D} & \text { aerodynamic drag force } \\ F_{L} & \text { aerodynamic lift force } \\ \mathrm{k} & \text { number of input parameters } \\ \mathrm{Ma} & \text { Mach number } \\ p & \text { pressure }\end{array}$

\section{INTRODUCTION}

Missiles or rockets are continuously developed for the defence industry by scientists and engineers. They are designed and improved according to missions. For example, some of them track to strike the movable enemy vehicles and some of them are

$\begin{array}{ll}S & \text { cross-sectional area } \\ \mathrm{q} & \text { heat flux vector } \\ u & \text { velocity of air in x direction } \\ v & \text { velocity of air in y direction } \\ w & \text { velocity of air in } \mathrm{z} \text { direction } \\ \tau & \text { viscous stress tensor } \\ \rho & \text { density of air } \\ y^{+} & \text {non-dimensional distance }\end{array}$

designed to strike a fix target or an area on the ground. Therefore, missiles are classified according to intended use such as air to air, air to ground, ground to air, ground to ground, guided/unguided or range of missile etc. The range of missile is crucial issue to reach the target or complete specified task exactly. As all air vehicles, aerodynamic 
performance is important in point of saving fuel or increasing range and stability of missile. Aerodynamic performance can be determined calculating lift to drag coefficients ratio.

Aerodynamic performance of a missile can be predicted performing wind tunnel test or using CFD method. However, wind tunnel test may be costly and required more time when compared with CFD solution. The solution can be validated with experimental data to obtain accurate results and after validation process, different scenarios can be solved using CFD method for saving time and cost. However, CFD method has some difficulties since solution equations are very complex. CFD software packages which use numerical solution technique make it possible to solve fluid flow problem within a reasonable amount of time for complex geometries. Some assumption can be accepted to solve fluid flow problem. Navier-Stokes Equation can solve some problems. However, some problems have complexity and Navier-Stokes Equation is not only sufficient. For instance; the separation, vortex eddies may form when the fluid flow is investigated at subsonic or supersonic speeds. Therefore, turbulent fluctuations effects should be taken into account and Reynolds stresses should be added to mean rate of deformation. Moreover, the Navier-Stokes equation may not give reasonable results for higher values of Reynolds number. These kinds of problems can be solved using appropriate turbulence model. Hence, the selection of the turbulence model is crucial issue to observe the boundary flow separation (Deck et al. 2002). Spalart-Allmaras, k-omega and k-epsilon turbulence models can be used to simulate the flow and selected one of them according to complexity geometry and flow speeds. In following paragraphs, previous studies related with flowfield computation and prediction of aerodynamic coefficients are mentioned.

CFD methods is used to compute flowfield and applied for different aerodynamic applications by engineers or scientists. Haider et al. (2017) performed efficient design of rotor blades in point of aerodynamics using CFD and the accuracy of the method was validated by comparing experimental results. Supreeth et al. (2020) also investigated the aerodynamics of S823 airfoil. For this, the airfoil was modified and computations were performed by means of SST k- $\omega$ turbulence model utilizing ANSYS Fluent. Another CFD simulation study was presented to increase aerodynamic performance reducing vorticity wake for $\mathrm{S} 833$ airfoil by $\mathrm{Yu}$ and Yang (2020). Numerical simulation was performed to investigate flapping wing aerodynamics by changing chord length by Kumar and Shah (2017). It was observed that dynamics of flapping wing was better understood thanks to this study.

The following paragraph is mentioned CFD solution and experimental study related with missile aerodynamics. Tahani et al. (2017) was proposed a study to improve aerodynamic efficiency by changing geometric parameters for a canard control missile. Lift to drag coefficients ratio were observed to decide performance of missile increasing the canard taper ratio and aspect ratio. Computational and experimental studies were performed to investigate wrap-around and flat fins of the missile rolling moment coefficients by Gülay et al (2011). The CFD calculation was carried out to find rolling moments and computational results were matched with experimental data. It was concluded that rolling moment coefficient of missiles with wrap-aroundfins is equal to the sum of the rolling moment coefficient of the flat-fins. Ryan (2011) performed aerodynamic analysis and optimization for morphing guided unpowered projectiles. The results of the study indicated that optimal geometry provided more range than baseline geometry. Smith (2009) focused on external ballistic problem for an axi-symmetric shape. In this study, two Euler solutions were used to solve projectile aerodynamics. The first method is Method of Characteristic (MoC) and second is finite volume numerical method. It was concluded that $\mathrm{MoC}$ solution did not show vorticity while finite volume method indicated the vorticity and shock wave on the nose and expansion region adjacent to the projectile. Sahu and Heavey (2017) studied on finned projectile to generate asymmetric pressure distribution. Using different geometric parameters, CFD analysis were performed to observe flow field and maximize the control of the projectile in terms of aerodynamics. An experimental study was proposed to investigate effect of canard shape on aerodynamic performance for Mach number of 0.5 by Guy et al. (1999). The efficiency of the canards evaluated based on pitching moments. It was inferred that static stability is reversely effected high aspect ratio, unswept, and untapered canards. Oktay et al. (2000) studied on two different missile geometries which are conventional and unconventional. In order to obtain aerodynamic coefficients, Euler solver code was improved and it reduce time cost. Silton (2005) focused on Navier-Stokes flow solver to compute the aerodynamic coefficients of a standard spinning projectile at different Mach numbers for subsonic to supersonic flows. The result of the study showed good agreement with the experimental results and semi-empirical aero prediction code. Zhang et al. (2013) proposed a study to predict aerodynamic coefficients at " $\mathrm{x} \pm$ " finned configurations for theater ballistic missile target. CFD methods and Missile DATCOM were used to calculate aerodynamic coefficients and compared with experimental study. The results of the study showed that the missile stability is quite good and provide requirements.

External shape design of flight vehicle is crucial issue in point of flight efficiency and performance. Therefore, shape optimization can be used to find optimum design for reduction cost and increasing efficiency. In the following paragraph, previous studies related with CFD based aerodynamic shape optimization methods are mentioned.

Yeong and Dol (2016) studied CFD based aerodynamic optimization to design a micro aerial vehicle. Moghadam and Jahangirian (2017) also performed aerodynamic optimization to control flow over transonic airfoil using Genetic Algorithm. Adeeb et al. (2016) proposed a study to improve aerodynamic efficiency of non-linear fan ceiling blades using Design of Experiment (DoE), Response 
Surface Method (RSM) and CFD. Another study related with aerodynamic optimization was performed to reduce flow loses of impeller optimizing wrap angle by Prabu et al. (2020).

In this paragraph, aerodynamic shape optimization for a missile or rocket is mentioned. Nobahari et al. (2006) presented an optimization study for a projectile. Two stochastic optimization methods that are Genetic Algorithm (GA) and Continuous Ant Colony System (CACS) were used. Nikbay et al. (2009) proposed an analysis and design work of an AGARD 445.6 wing to maximize the lift-to-drag ratio and to minimize the total weight. Anderson et al. (2000) proposed also optimization study for missile aerodynamic using Pareto genetic algorithm. Misaghian et al. (2007) performed shape optimization improving a code to calculate aerodynamic heating for the fins. Tanil et al. (2009) proposed a paper for optimization of a missile design using MATLAB with Genetic Algorithm. Dyer et al. (2012) also proposed to aerospace engineering design using real coded Genetic algorithm. Vidanovic et al. (2017) focused on Multidisciplinary design optimization (MDO) for external missile configuration. CFD solution and experimental study were performed to find drag and lift coefficients at different supersonic speeds and angles of attack. Ageev and Pavlenko (2016) proposed a paper for reduction drag coefficient using Euler and Reynolds averaged Navier-Stokes (RANS) equations. SearsHaack body of revolution shape was improved making front part of the body blunted. It was concluded that aerodynamic drag decreased about 20 $\%$ when compared with Sears-Haack body. Runduo and Xiaobing (2018) performed aerodynamic shape optimization for a rocket. Non-dominated sorting genetic algorithm (NSGA-II) and the real coding genetic algorithm (RGA) were used and the results of the study showed that the lift-to-drag ratio was increased and the maneuverability was improved as a result of optimization process. Munoz et al. (2015) proposed a study related with aerodynamic shape optimization using Adjoint methods for train nose shape. The drag coefficient was reduced about $7 \%$. Aelaei et al. (2019) also proposed optimization study using CFD based response surface method for delta wing. The results of study showed that lift to drag ratio increased about $28 \%$ when compared with benchmark. Sumnu et al. (2020) proposed a shape optimization study for a missile using GA. The results showed fins of missile were an effective factor on the aerodynamic performance and lift to drag ratio was improved thanks to optimization algorithm.

In this study, the aim is to investigate wing effect on aerodynamic performance since there are few studies related to investigation of wing effect on missile aerodynamics at subsonic and supersonic speeds. At first step, the CFD modelling technique is validated against literature based experimental data. Then the wings are mounted to the selected missile model and CFD solution of modified missile model is performed to determine aerodynamic performance using SST k- $\omega$ turbulence model. Optimization study is carried out for mounted wing to improve lift to drag ratio. Seven design parameters are used to optimize for wing geometry. In addition, the response surface analysis is presented to show which design variable of mounted wing is more effective on results. The following section, missile geometry and mesh generation are explained.

\section{MisSILE GEOMETRY AND MESH GENERATION}

Missile geometry and computational fluid domain are generated using Designmodeler in ANSYS. The missile with tailfin configuration selected from previous study (Vidanović et al. 2017) is presented in Fig. 1. Wing is then mounted to the selected missile. The mounted wing dimensions are given in Fig. 2 and modified missile geometry is presented in Fig. 3.

Mesh generation is implemented using Mesh in ANSYS. The prismatic mesh (structured) is generated around the missile wall to capture flow field and separation of flow. The boundary layer of the missile is formed with 25 prismatic layers and 1.2 grow rate. Tetrahedral mesh (unstructured) mesh is formed for remaining part of the computational fluid domain using the patch conforming method. The mesh generation of the missile wing is presented in Fig. 4 and mesh generation of missile body is presented in Fig. 5.

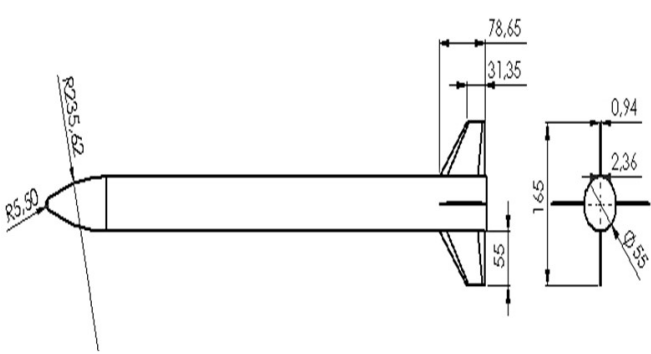

Fig. 1. Selected missile with tailfin configuration (Vidanović et al. 2017).

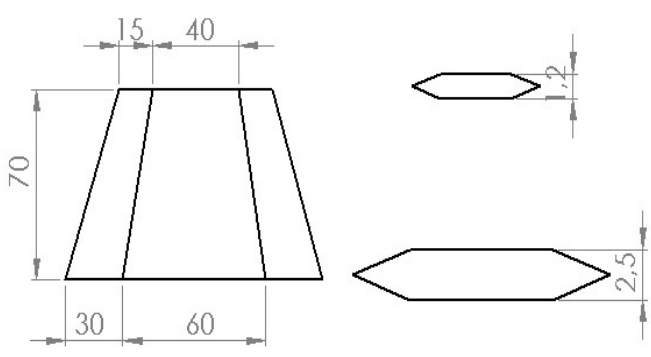

Fig. 2. Mounted wing dimensions.

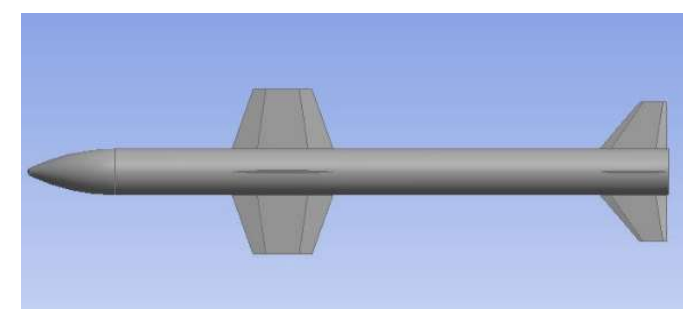

Fig. 3. Missile with wing and tailfin configuration. 


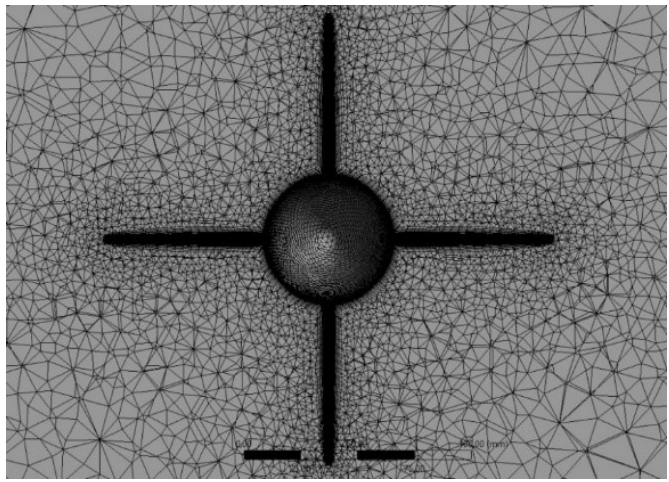

Fig. 4. Mesh generation around the wing missile.

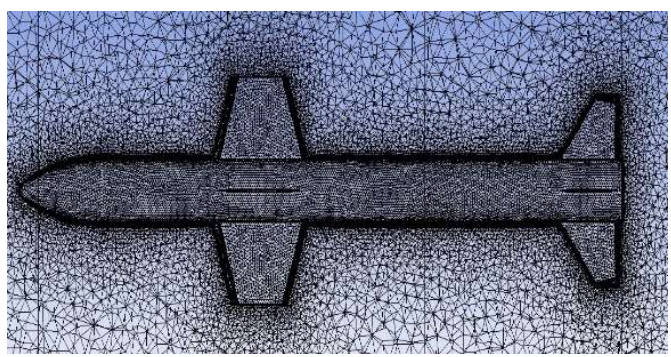

Fig. 5. Mesh generation missile body.

The solution is firstly performed on a courser mesh with the first cell non-dimensional wall distance $\mathrm{y}^{+} \approx$ $20-100$ and the solution is continue generating finer mesh with the first cell non-dimensional wall distance $\mathrm{y}^{+} \approx 0,9-8$. Moreover, mesh independency is crucial to show correct solution and mesh generation methods. Hence, efficient mesh number should be determined to find reasonable amount of solution time. CFD solutions are implemented by generating eleven different mesh numbers that are between about 300000 and 3.7 million. The solution results are showed that the efficient mesh number is 2341035 since the solution result is negligible change more than 2341035 mesh number elements. In addition, the convergence of the calculated aerodynamic coefficients and residuals are provided with generated mesh structure and mesh number. Figure 6 represents mesh independency for the lift coefficient $\left(\mathrm{C}_{L}\right)$ versus number of mesh elements.

\section{Computational Approach}

Numerical simulation plays an important role in terms of understanding the flow physics. In this study, CFD software package Fluent is used to carry out solutions. Finite volume method is used by Fluent code for solution of conservation equations. In order to predict of air flow, Navier-Stokes equations are used. In this study, 3-D, steady-state, compressible flow is analyzed.

In this study, computational fluid domain is large enough to perform solution of missile flow field. It is formed as cylinder and its length is 20 times the length of missile body and the radius of cylinder is 10 times length of missile body. The missile body is defined as a wall type because of no-slip condition.
This boundary condition is more convenient in point of consuming of computational time and resource.

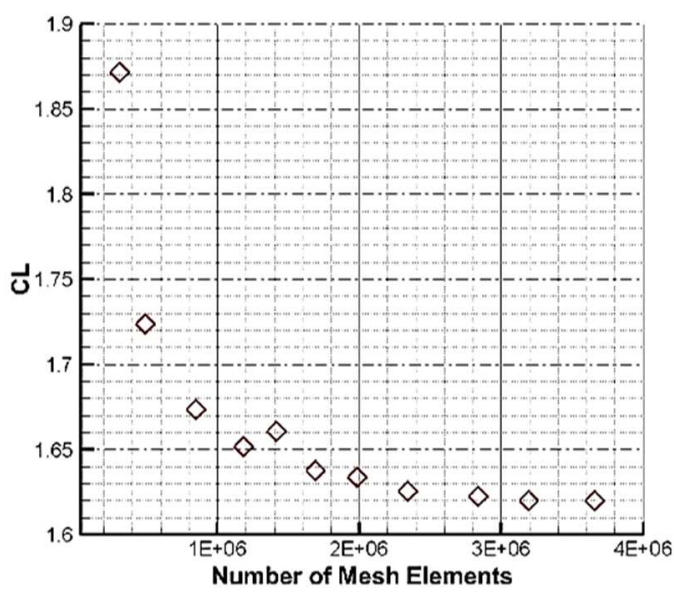

Fig. 6. Mesh Independency Chart.

In order to solve flow field, SST k- $\omega$ turbulence model is chosen since it is suitable to solve complex aerodynamic shape and shows superior performance at subsonic and supersonic flows. This turbulence model includes both $\mathrm{k}-\omega$ and $\mathrm{k}-\varepsilon$ turbulence models. In the inner region of the boundary layer, the $k-\omega$ model is used. In outer region, $\mathrm{k}-\omega$ model switches to standard k- $\varepsilon$ model (Menter 1994). Therefore, this model gives quite accurate results near the wall and in case of flow separation and circulation. The SST $\mathrm{k}-\omega$ model is utilized to obtain accurate result in some previous studies that is related with missile aerodynamics (Vidanović et al. 2014).

The solver type is defined as steady-state and density based (since flow is compressible) for computing the flowfield. For solution method and gradient, the implicit formulation with Roe-FDS flux type and the least square cell based are selected. The second order upwind is selected for flow (Ansys Inc. 2009). The Courant number that controls over the time-stepping scheme is determined between the value of 0.7 and 1 for all solutions.

The change of flow residuals and aerodynamic coefficients are observed during the computations. If flow residuals reach to $10^{-5}$ and the value of aerodynamic coefficient is not change greater than $1 \%$ during 100 iterations, the solution is finished. The convergence occurs when the number of iterations is between 900 and 1200 .

The continuity, momentum and energy equations are solved by the fluid solver. The whole system of governing equations is given by Eq. (1) (Anderson and Wendt 1995).

$$
\frac{\partial}{\partial t} \vec{U}+\frac{\partial}{\partial x} \vec{F}+\frac{\partial}{\partial y} \vec{G}+\frac{\partial}{\partial z} \vec{H}=0
$$

$\vec{U}, \vec{F}, \vec{G}$ and $\vec{H}$ are defined as; 
$\vec{U}=\left\{\begin{array}{c}\rho \\ \rho u \\ \rho v \\ \rho w \\ \rho E\end{array}\right\}, \vec{F}=\left\{\begin{array}{c}\rho u \\ \rho u^{2}+p-\tau_{x x} \\ \rho v u-\tau_{x y} \\ \rho w u-\tau_{x z} \\ \rho u E+p u-q_{x}-u \tau_{x x}-v \tau_{x y}-w \tau_{x z}\end{array}\right\}$

$\vec{G}=\left\{\begin{array}{c}\rho v \\ \rho u v-\tau y x \\ \rho v^{2}+p-\tau y y \\ \rho w v-\tau y z \\ \rho v E+p v-q_{y}-u \tau y x-v \tau_{y y}-w \tau y z\end{array}\right\}$

$\vec{H}=\left\{\begin{array}{c}\rho w \\ \rho u w-\tau_{z x} \\ \rho v w-\tau_{z y} \\ \rho w^{2}+p-\tau_{z z} \\ \rho w E+p w-q_{z}-u \tau_{z x}-v \tau_{z y}-w \tau_{z z}\end{array}\right\}$

$\vec{F}, \vec{G}$ and $\vec{H}$ are the called the flux terms and the solution vector is $\vec{U}$.

\section{RESUlts OF CFD Simulation}

In this study, CFD solution is performed at supersonic Mach number and $6^{\circ}$ AoA using selected missile model that has experimental test data (Vidanović et al. 2017). The results are observed that experimental results and CFD solutions are matched with each other. Figure 7 represents the drag coefficients for CFD solutions and experimental results versus Mach numbers at $6^{\circ}$ AoA. Lift coefficients and experimental results versus Mach number at $6^{\circ} \mathrm{AoA}$ is presented in Fig. 8.

CFD solution of modified missile is performed at $6^{\circ}$ AoA and both at subsonic and supersonic speeds after validation is provided for selected missile designed with tailfin configuration. The solution results of modified missile showed that aerodynamic performance is better than missile with tailfin configuration. Aerodynamic coefficients $\left(\mathrm{C}_{\mathrm{L}}\right.$ and $\mathrm{CD}_{\mathrm{D})}$ and improvement ratio of both missiles are presented in Table 1.

Pressure contour is important to observe pressure values occurring on missile geometry and show flow physics. In this study, in order to show effect of the wing, the pressure contours are presented in Fig. 9 and Fig. 10 for both modified and baseline missile models at 1.4 and 0.8 Mach numbers, respectively. When examined the Fig. 9, the shock waves can be observed due to supersonic flow. In addition, the lower pressure contours occurs at trailing edge of wing and rear of the missile body. This can cause increase the drag forces however, lift force occurring on the wings more than increase of drag force. Thus, lift to drag ratio is improved when compared baseline missile model. The shockwave doesn't observe in Fig. 10 but lower pressure occur some area on missile body at 0.8 Mach number for both missile models. Lift force increase thanks to wing configuration however, it could not be observed from pressure contours in Fig. 10. Therefore, velocity streamlines can help to understand flow physics for wing performance.

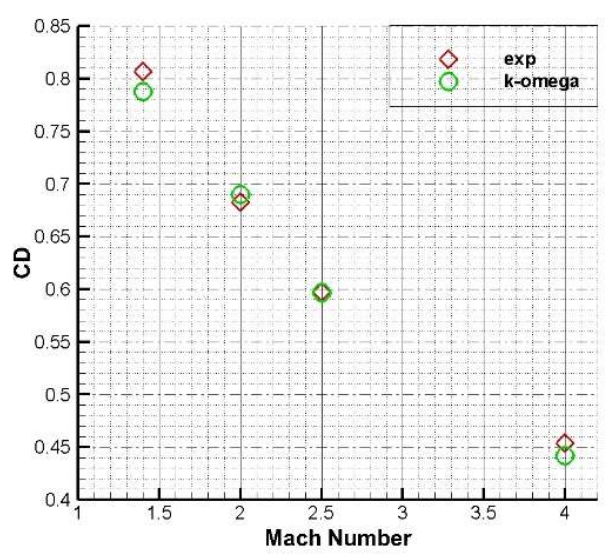

Fig. 7. Drag coefficients versus Mach number at $6^{\circ}$ AoA.

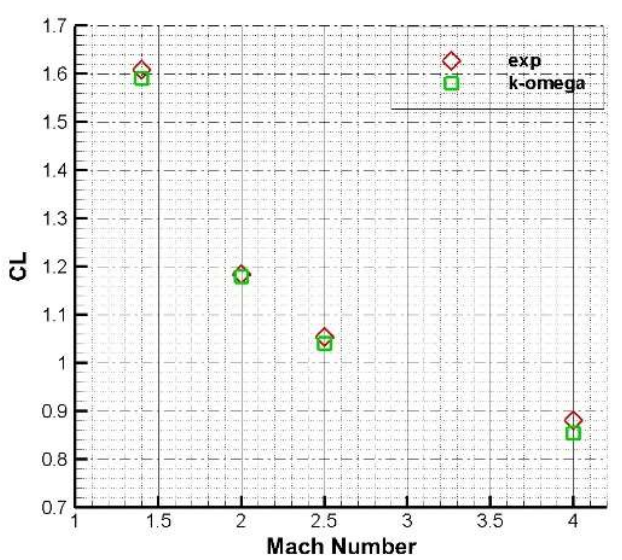

Fig. 8. Lift coefficients versus Mach number at $6^{\circ}$ AoA.

Table 1 Comparison missile with tailfin and modified missile configurations.

\begin{tabular}{|c|c|c|c|}
\hline \multicolumn{2}{|c|}{ Ma } & 0.8 & 1.4 \\
\hline Missile with tailfin & $C_{L}$ & 0.821 & 1.547 \\
\cline { 2 - 4 } & $C_{D}$ & 0.454 & 0.797 \\
\hline Modified Missile & $C_{L}$ & 1.572 & 3.232 \\
\cline { 2 - 4 } & $C_{D}$ & 0.622 & 1.156 \\
\hline Improvement $C_{L} / C_{D}(\%)$ & 39.77 & 44.15 \\
\hline
\end{tabular}

Velocity streamline can give information about flow physics. Therefore, velocity streamlines of missile wing and tailfin are presented in Fig. 11 and Fig. 12 for 0.8 and 1.4 Mach numbers, respectively. Flow separation and vortex could not be observed on wing when examined the velocity streamline in part (a) of Fig. 11. However, vortex has been observed on the tailfins at 0.8 Mach number. Hence, wings provided 
contribution for lift force and improved aerodynamic performance. Similarly, when examined the velocity streamline at 1.4 Mach number, the separation could not be observed on the wing but vortex occurred near missile body. The velocity streamlines of wings are more uniform according to air flow of tailfins and this provides higher lift force. This also demonstrates that the wings improve the missile aerodynamic performance when compared with missile with tailfin configuration.

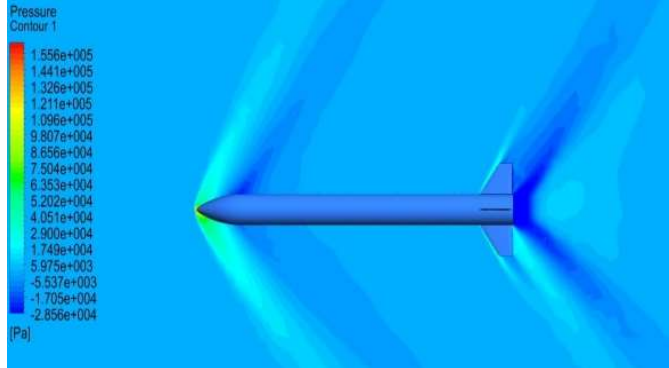

(a) Baseline missile model

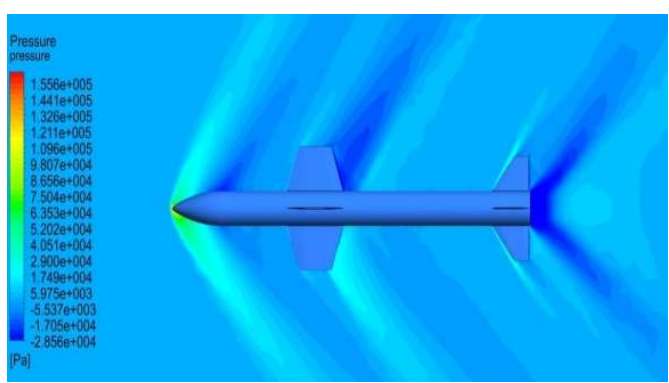

(b) Modified missile model

Fig. 9. Pressure Contour at 1.4 Mach number and $6^{\circ}$ AoA.

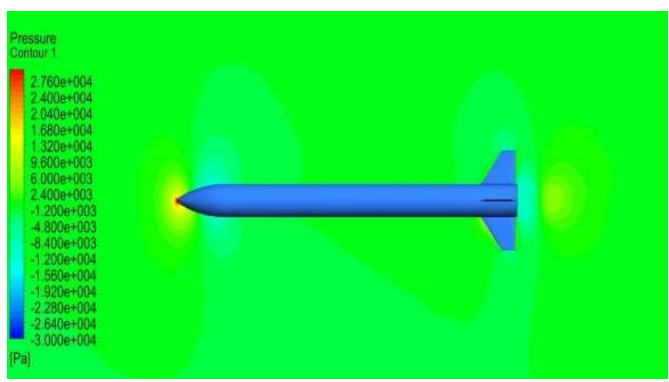

(a) Baseline missile model

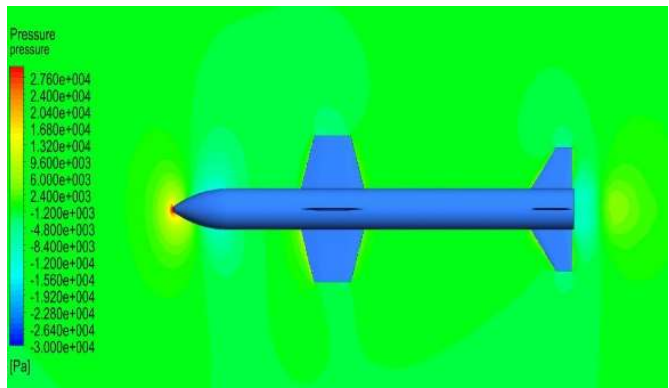

(b) Modified missile model

Fig. 10. Pressure Contour at 0.8 Mach number and $6^{\circ} \mathrm{AoA}$.

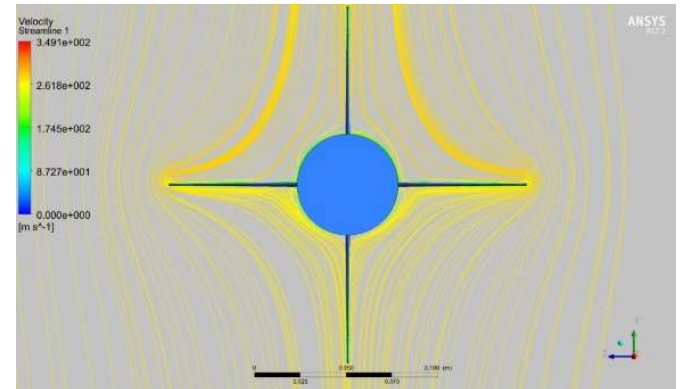

(a) Wing geometry

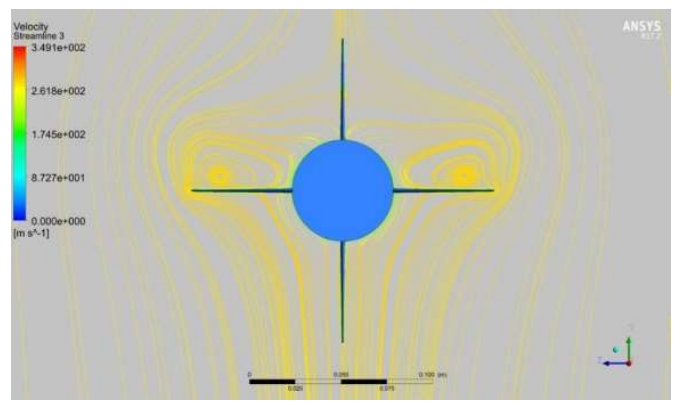

(b) Tailfin geometry

Fig. 11. Velocity streamlines for 0.8 Mach number.

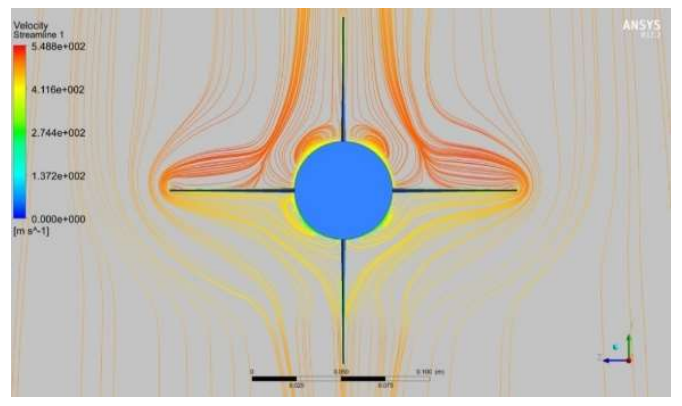

(a) Wing geometry

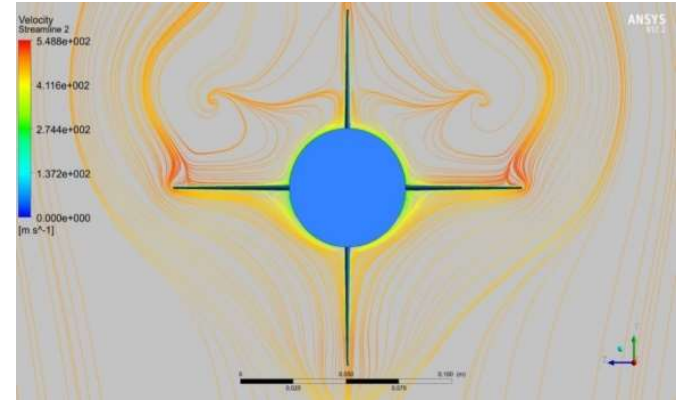

(b) Tailfin geometry

Fig. 12. Velocity streamlines for 1.4 Mach number.

\section{Multi-ObJective Genetic ALGORITHM OPTIMIZATION}

Genetic algorithms (GA) are types of Evolutionary Algorithm (EA) that is commonly used to generate optimum solution and search problems. The Genetic algorithms were described by Holland (1975). Goldberg (1989) also developed the Genetic 
Algorithm. GA is a stochastic search approach and depends on principles of natural selection and genetics. They are widely used to solve engineering problems and industrial applications since it can find good results and perform the study in reasonable amount of time. In addition, it can be stated that the solution results for optimization problems are quite accurate and they are more effective method for external shape optimization when literature related with genetic algorithms is examined (Tanil et al. 2009).

In GA, chromosomes are defined as a solution vector $\mathrm{x} \in \mathrm{X}$ and chromosomes are collected to create population. The chromosomes in the population are evaluated and filtered using some measure of fitness. GA involves two operators that are mutation function and crossover function to generate new chromosomes that are called offspring. Crossover process is used to generate new chromosomes by combining two chromosomes. Using these operators, a new population is obtained and it replaces with old population. It generates good generation according to the fitness values. The process is continued to find best chromosome that presents the optimum solution of problem (Gen and Cheng 1997).

In engineering problems, multi-objective optimization is generally used since the problems are complex and objective is generally more than one. GA is convenient to use for multi-objective optimization problem because it can be modified to obtain a set of multiple solutions in a single run. In addition, diverse set of solution can be found in different regions by means of GA. GA can use good solutions to generate non-dominated solutions in unknown parts of the Pareto front in crossover process. Hence, it can be stated that GA is appropriate to use for multi-objective optimization design (Konak et al. 2006).

In this study, the aim is to improve wing of missile applying optimization technique since there are few study in literature that especially investigate the wing effects and perform optimization for aerodynamic performance. For this purpose, Multi-Objective Genetic Algorithm (MOGA) is used to solve optimization problem utilizing ANSYS DesignXplorer. The following sub-sections are mentioned about optimization algorithm set up.

\subsection{Mathematical Representation of the Multi-objective Optimization}

A Multi-Objective optimization problem can be expressed as follows: (Deb 1999; Marler and Arora 2004).

$$
\begin{array}{lll}
\text { Maximize: } & f(x)=\left[f_{1}(x), f_{2}(x), \ldots f_{n}(x)\right] \\
\text { Subject to: } & g_{j}(x) \leq 0 & j=1,2 \ldots m \\
& h_{k}(x)=0 & k=1,2 \ldots q \\
& x_{i}^{l} \leq x_{i} \leq x_{i}^{u} & i=1,2 \ldots p
\end{array}
$$

Where $\mathrm{x}=\left[\mathrm{x}_{1}, \mathrm{x}_{2}, \ldots . \mathrm{x}_{\mathrm{j}}\right]^{\mathrm{T}}$ is the vector of design variables, $f(x)$ is the multi-objective vector, $f_{n}(x)$ is the objective function, $\mathrm{g}_{\mathrm{j}}(\mathrm{x})$ and $\mathrm{h}_{\mathrm{k}}(\mathrm{x})$ are the constraints and $x_{i}^{1}$ and $x_{i}{ }^{u}$ are lower and upper bounds of the design variables, respectively.

\subsection{Objective Function}

The missile aerodynamic performance can be improved increasing lift to drag ratio. Therefore, $C_{L}$ and $C_{D}$ are crucial to determine the performance. In the study, these aerodynamic coefficients are considered objective function (output function) for solution of optimization problem.

\subsection{Design Variable}

The modified missile geometry involves four main parts which are wing, tailfins nose, and body. These components are important to specify aerodynamics characteristic for missile. However, in this study, missile wing is only investigated and optimized. The selection of design variables is crucial for optimization solution. In our study, 7 parameters that are wing root chords $\left(\mathrm{L}_{\mathrm{wr}}\right)$, wing tip chords $\left(\mathrm{L}_{\mathrm{wt}}\right)$, wing root edge length $\left(\mathrm{L}_{\mathrm{re}}\right)$, wing tip edge length $\left(\mathrm{L}_{\mathrm{te}}\right)$, root $\left(\mathrm{T}_{\mathrm{r}}\right)$ and tip $\left(\mathrm{T}_{\mathrm{t}}\right)$ thickness of wing and leading-edge and trailing edge $(\gamma)$ sweepbacks are selected for optimization of the missile wing geometry. The design variables show in Fig. 13 for missile wing. Lower boundary (LB), upper boundary (UB) and baseline values of the wing design variables for the optimization solution are presented in Table 2.

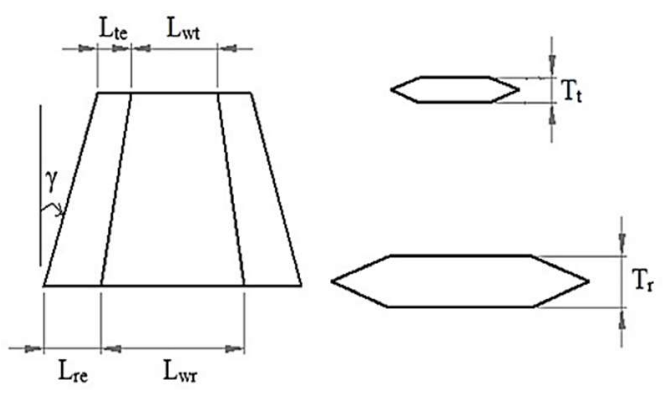

Fig. 13. Wing design variables.

\subsection{Constraints}

Design parameters or objective functions may include some limitations when optimization problems are solved. Thus, the optimization results should provide the limitations and requirements for specified problem. The upper and lower values of design variables are determined in Table 2. In addition, lift coefficient for baseline missile should be smaller than optimum missile geometry. The other constraint is drag coefficient and its value for baseline missile geometry should be greater than optimum missile geometry. The following notations are presented to define optimization problem. Equation (2) and Eq. (3) represent the lift and drag coefficients.

Maximize: 
Table 2 Baseline, lower and upper values of the wing design variables

\begin{tabular}{|c|c|c|c|}
\hline $\begin{array}{c}\text { Design } \\
\text { Variables }\end{array}$ & Base & LB & UB \\
\hline $\mathbf{L}_{\mathbf{w r}}(\mathbf{m m})$ & 60 & 45 & 75 \\
\hline $\mathbf{L}_{\mathbf{w t}}(\mathbf{m m})$ & 40 & 25 & 55 \\
\hline $\mathbf{L}_{\mathbf{r e}}(\mathbf{m m})$ & 30 & 20 & 40 \\
\hline $\mathbf{L}_{\mathrm{te}}(\mathbf{m m})$ & 15 & 7.5 & 22.5 \\
\hline $\mathbf{T}_{\mathbf{r}}(\mathbf{m m})$ & 3 & 1 & 5 \\
\hline $\mathbf{T}_{\mathbf{t}}(\mathbf{m m})$ & 1.5 & 0.5 & 2.5 \\
\hline$\gamma(\mathbf{d e g})$ & $19.65^{\circ}$ & $10^{\circ}$ & $30^{\circ}$ \\
\hline
\end{tabular}

$C_{L \max }(x)=\frac{F_{L}}{\frac{1}{2} \rho u^{2} S}$

Minimize:

$C_{D \min }(x)=\frac{F_{D}}{\frac{1}{2} \rho u^{2} S}$

With respect to:

$\mathrm{X}$ is optimization variables vector (Design variables are presented in Table 2 that shows upper and lower value of geometric parameters)

Subject to:

$C_{D}($ opt $) \leq C_{D}$ (base)
$C_{L}($ opt $) \geq C_{D}$ (base)

\subsection{Optimization Solution}

In the optimization process, the problem should be solved by providing specified constraints and using determined design variables for missile wing. For this purpose, CFD solution is performed to calculate objective functions at 0.8 and 1.4 Mach numbers and $6^{\circ}$ AoA in Fluent ANSYS. The SST k- $\omega$ turbulence model is selected for solutions. Optimization solution is then performed using Ansys DesignXplorer which is an integrated Ansys Workbench to perform parametric analyses.

In the beginning of optimization step, modified missile geometry is formed Design Modeler. Specified design variables are chosen for transformation of Parameter set block of the ANSYS Workbench. Mesh is then generated using ANSYS Mesh. After mesh generation is completed, ANSYS Fluent is used to perform CFD solution. Drag and lift coefficients are computed according to initial and boundary conditions. In order to gather input and output values, computed value of aerodynamic coefficients are transferred to parameter set block. The gathered parameters are used in ANSYS DesignXplorer for optimization process. Design points are generated in the first step of DesignXplorer.

In this study, Central Composite Design (CCD) is used to generate design points (DP). Using 7 design variables, 79 design points are generated in ANSYS Design of Experiment (DoE). Hence, CFD solution of constructed missile should be repeated 79 times for each design point. CCD is first proposed by Box and Wilson (1951). The calculation of design points for CCD is defined as following Eq. (4).

$D P=2^{(k-f)}+2 k+1$

Where $\mathrm{k}$ represents the number of input parameters, $\mathrm{f}$ is factorial which a quantity to limit the design points. This equation is used to find reasonable number of design points and it provides to escape excessive number of design points for surrogate model.

Response surface is performed with respect to the obtained data in second step. Kriging model is used to generate response surface model. After that, parameters and design points are used to run optimization process using MOGA. In the optimization process, configuration is generated using 100 samples and 100 samples per iteration. Then, CFD are performed to verify the solutions for obtained 3 candidate results. One of candidates results are selected as optimum solution. The convergence is reached after 781 and 974 computations for 0.8 and 1.4 Mach numbers, respectively.

\subsection{Response Surface Analysis}

Response surface are functions that define the output parameters in point of the input parameters. Response surface methodology is to use a sequence of designed experiments for obtaining an optimal response. Combining this methodology in design optimization is called as Response Surface Optimization (RSO). This methodology was firstly described by Box and Wilson (1951).

Response surface provides to obtain the approximated values of output parameters that are analyzed in design space without the need to carry out a complete solution. Genetic aggregation and Kriging Response Surface types are widely used to analyze design space. Genetic Aggregation is generally used for high number of design points while Kriging type is efficient in a large number of cases and appropriate for highly nonlinear responses. Kriging model is used for approximating deterministic computer model. (Hao et al. 2010). Hence, in this study, Kriging type is determined to perform Response Surface analysis due to gives more accurate results for nonlinear responses. The general statement of Kriging model is given Eq. (5)

$$
\begin{aligned}
& y(x)=\sum_{l=1}^{p} \beta_{l} f_{l}\left(x_{i}\right)+z\left(x_{i}\right)=\mathrm{f}^{T}\left(x_{i}\right) \beta+z\left(x_{i}\right), \\
& i=1,2, \ldots, n
\end{aligned}
$$

Where $\beta$ is the coefficient vector, $\mathrm{f}(\mathrm{x})$ is the polynomial vector, $\mathrm{n}$ is the number of sample points, $\mathrm{p}$ is the number of $f_{l}(x), \mathrm{z}(\mathrm{x})$ indicates the error.

Response surface chart for the drag force with tip chords and root chords of wing at 1.4 Mach number and $6^{\circ}$ AoA is presented in Fig. 14. It can be observed that the wing tip chord is more effective than wing root chord and the increase of wing chord 


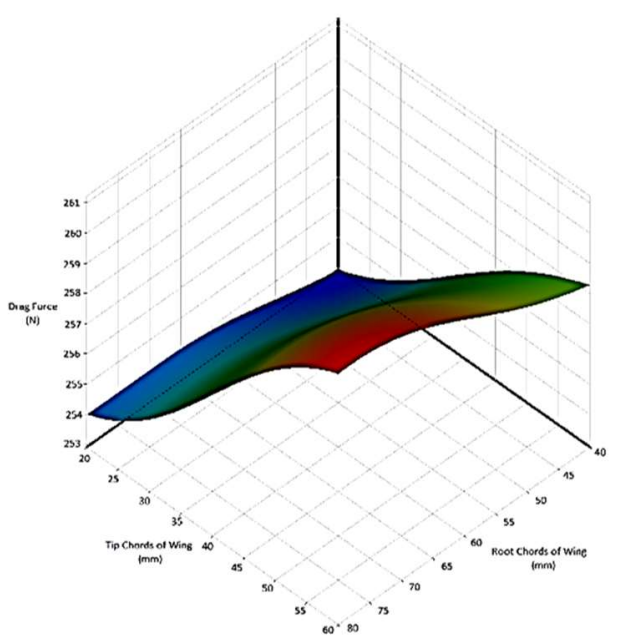

Fig. 14. Response surface for drag force with tip chords and root chords of wing at 1.4 Mach number and $6^{\circ}$ AoA.

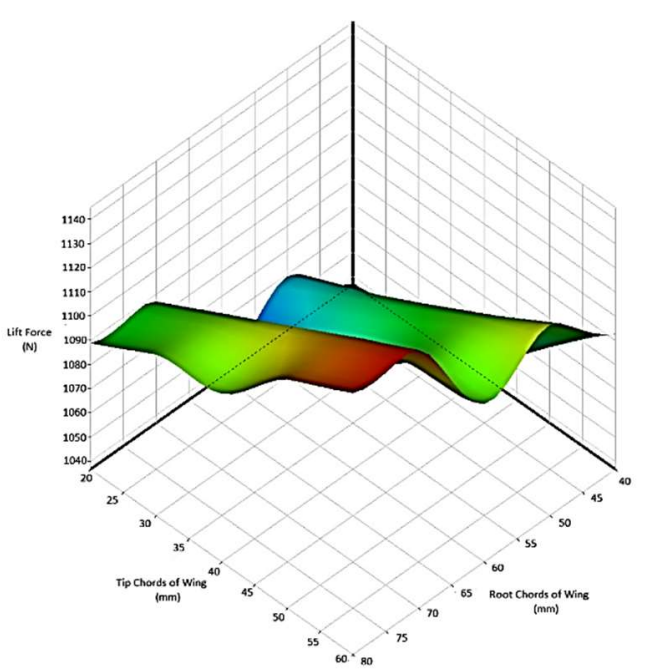

Fig. 15. Response surface for lift force with tip chords and root chords of wing at 1.4 Mach number and $6^{\circ}$ AoA.

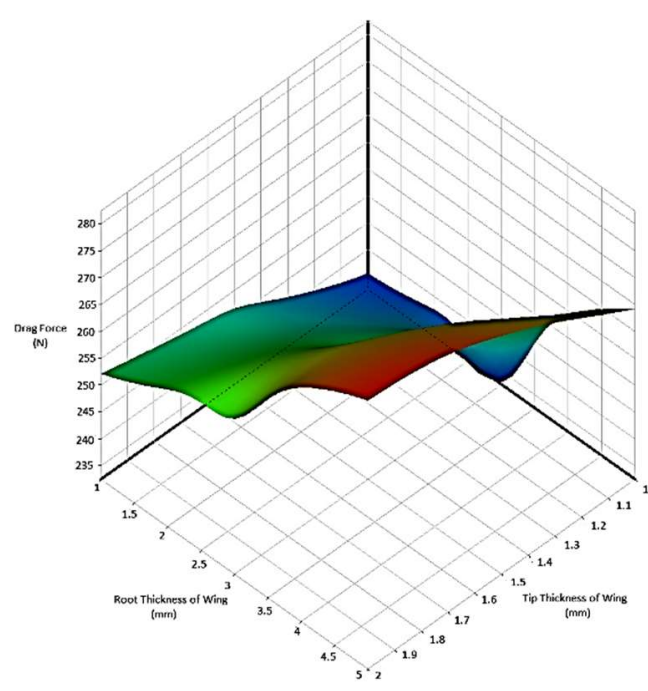

Fig. 16. Response surface for drag force with root thickness and tip thickness of wing at 1.4 Mach number and $6^{\circ}$ AoA.

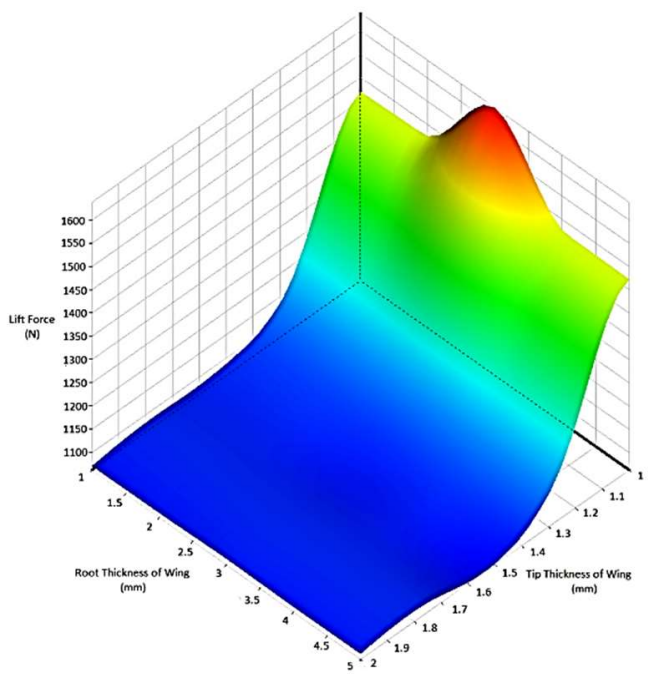

Fig. 17. Response surface for lift force with root thickness and tip thickness of wing at 1.4 Mach number and $6^{\circ}$ AoA.

is caused increase of drag force. However, when examine the Fig. 15 that shows lift force with tip chords and root chords of wing, they provide positive effect for lift force. It is concluded that lift force increase rate is more than drag increase rate.

Tip and root thickness of wing is also investigated for drag and lift forces at 1.4 Mach number and $6^{\circ}$ AoA which are presented in Fig. 16 and Fig. 17, respectively. It can be observed that drag force increase when tip and root chords length is increase since cross-sectional area increase. It can be understood that lift force suddenly decrease when tip thickness increase. This reason may be due to flow separation when thickness is increase.

The 2-D charts are also presented to show response surface model and design points in Fig. 18 and Fig. 19 due to show accuracy and convenience of presented response surface model. When observed these figures, it can be stated that the kriging model improves the response surface model since it apparently provides the non-linearities (RojasSolórzano 2017).

\subsection{Optimization Results}

The improvement of lift and drag coefficients are achieved after optimization process according to results. The aerodynamic performance is determined with the value of lift to drag ratio. The aerodynamic coefficients and improvement of their ratio are presented in Table 3. Design variables of optimum modified missile are also given in Table 4 . Wing thickness and length decrease when compared with baseline wing geometry. In addition, the surface area of missile wing for supersonic flow is higher than surface area for subsonic flow. Finally, it can be said that optimum results more efficient in terms of reduction of material and performance. 


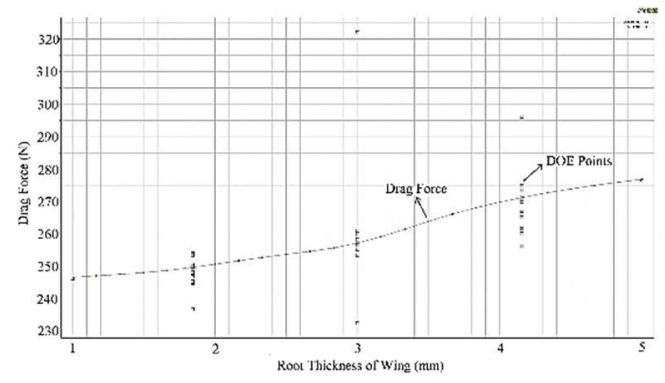

Fig. 18. 2-D Response surface for drag force versus root thickness of wing with DOE points.

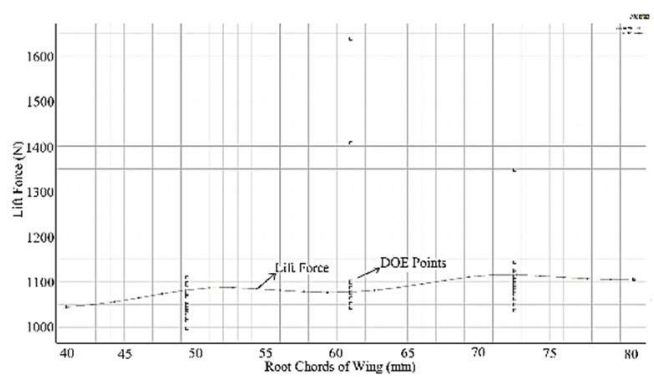

Fig. 19. 2-D Response surface for lift force versus root chords of wing with DOE points.

Table 3 Optimization Results

\begin{tabular}{|c|c|c|}
\hline Ma Number & $\mathbf{0 . 8}$ & $\mathbf{1 . 4}$ \\
\hline $\mathbf{C}_{\mathbf{L}}$ Base & 1.572 & 3.232 \\
\hline $\mathbf{C}_{\mathbf{L}}$ Opt. & 1.603 & 3.308 \\
\hline $\mathbf{C}_{\mathbf{D}}$ Base & 0.622 & 1.156 \\
\hline $\mathbf{C}_{\mathbf{D}}$ Opt. & 0.589 & 1.105 \\
\hline $\begin{array}{c}\text { Improvements } \\
\mathbf{C}_{\mathbf{L}} / \mathbf{C}_{\mathbf{D}}(\%)\end{array}$ & 7.479 & 7.015 \\
\hline
\end{tabular}

Table 4 Optimum Design Variables

\begin{tabular}{|c|c|c|c|}
\hline $\begin{array}{c}\text { Design } \\
\text { Variables }\end{array}$ & Base & $\begin{array}{c}\text { Opt. } \\
\text { 0.8 Ma } \\
\text { number }\end{array}$ & $\begin{array}{c}\text { Opt. } \\
\mathbf{1 . 4} \text { Ma } \\
\text { number }\end{array}$ \\
\hline $\mathbf{L}_{\mathbf{w r}}(\mathbf{m m})$ & 60 & 46.279 & 59.748 \\
\hline $\mathbf{L}_{\mathbf{w t}}(\mathbf{m m})$ & 40 & 28.725 & 29.433 \\
\hline $\mathbf{L}_{\mathbf{r e}}(\mathbf{m m})$ & 30 & 26,665 & 35.111 \\
\hline $\mathbf{L}_{\text {te }}(\mathbf{m m})$ & 15 & 18.64 & 15.601 \\
\hline $\mathbf{T}_{\mathbf{r}}(\mathbf{m m})$ & 3 & 1.2567 & 1.20 \\
\hline $\mathbf{T}_{\mathbf{t}}(\mathbf{m m})$ & 1.5 & 1.015 & 1.006 \\
\hline $\boldsymbol{\gamma} \mathbf{( d e g})$ & 19.65 & $13.49^{\circ}$ & $26.35^{\mathbf{o}}$ \\
\hline
\end{tabular}

Pressure distribution helps to observe the improvement wing aerodynamics and understand differences between base and optimum case of missile wing. Therefore, pressure distributions of modified missile with specific locations are presented in Fig. 20 and Fig. 21 for both optimum and baseline geometry at 0.8 and 1.4 Mach numbers, respectively. It can be stated that pressure difference between lower and upper surface of optimized missile wing are more than base missile wing when examined between $0.4 \mathrm{~m}$ and $0.6 \mathrm{~m}$ on $\mathrm{x}$-coordinate in Fig. 20. Moreover, maximum pressure takes places about leading edge of the base wing model and it is greater than optimum model. Thus, drag force that occurs on base wing is greater since pressure difference between leading and trailing edge of base wing model is higher than optimum one. When observed Fig. 21 at points between $0.5 \mathrm{~m}$ and $0.54 \mathrm{~m}$ on $\mathrm{x}$-coordinate, the pressure acting on the leading edge of optimum wing reduce compared with base wing. In addition, pressure value on trailing edge for optimum and base wings are approximately same. Hence, pressure differences of optimum wing between leading and trailing edges is smaller than base wing for solution at 1.4 Mach number. This is the reason why optimum model shows better performance. Furthermore, pressure difference is negligible change around the tailfin of missile. This means that wing does not adversely affect the air flow around the missile tailfin for subsonic and supersonic flows. Hence, it can be said that mounted wing improves the selected missile aerodynamics.

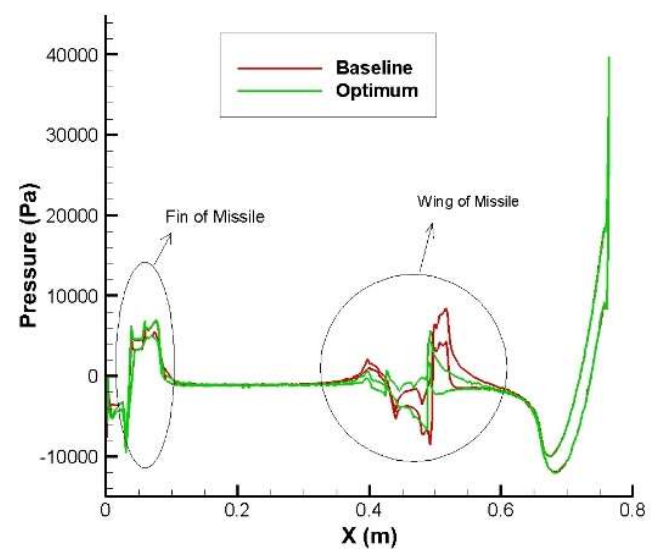

Fig. 20. Pressure distribution of modified missile at 0.8 Mach number and $6^{\circ} \mathrm{AoA}$.

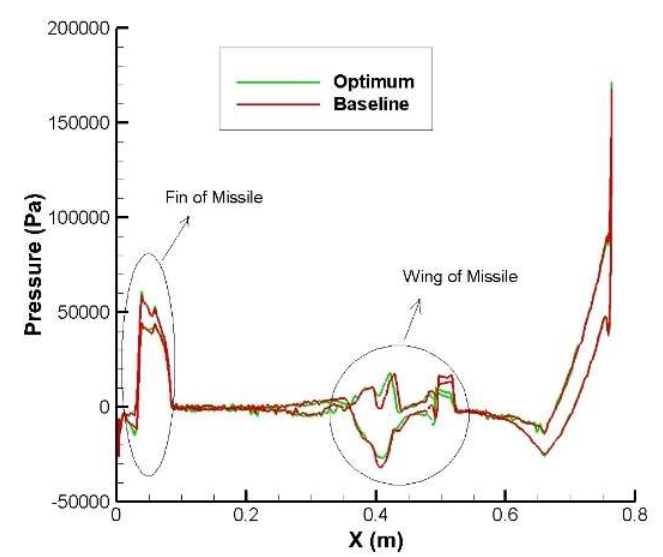

Fig. 21. Pressure distribution of modified missile at 1.4 Mach number and $6^{\circ} \mathrm{AoA}$.

\section{CONCLUSION}

In this study, CFD simulation technique with SST $\mathrm{k}$ - $\omega$ turbulence model was used to validate 
experimental results of missile model reported in the literature study. The validation was achieved and showed by comparing with experimental results. Then the selected missile model was modified to improve aerodynamic performance by mounting wings on the body. In order to find aerodynamic coefficients of modified missile model, CFD solution was repeated using SST k- $\omega$ turbulence model at 0.8 and 1.4 Mach number and $6^{\circ}$ AoA in ANSYS Fluent. The results of solution was indicated that lift to drag coefficient ratio improves about \% 39.77 and \% 44.15 at 0.8 Mach and 1.4 Mach numbers, respectively when the results were compared with missile with tailfin configuration. In addition, mounted wing was optimized to improve further aerodynamic performance. In optimization process, MultiObjective Genetic Algorithm (MOGA) was used since the algorithm is more convenient for multiobjective optimization solution. Lift and drag coefficients were selected as objective function and seven wing parameters were determined as design variables. Response surface analysis was presented to show which design parameters are more effective on drag and lift coefficients. It can be said that the wing thickness was more effective than wing chords for drag force and the tip thickness of wing was more effective on lift force when response surface charts were examined. After optimization was performed, it can be stated that lift to drag ratio increased about $\% 7.47$ and \% 7.015 at 0.8 and 1.4 Mach numbers, respectively. In addition, pressure distributions that show pressure differences between optimum and baseline of modified missile model were presented to observe optimization results better. Finally, it can be said that optimum results are quite efficient in terms of aerodynamic performance since modified and optimized missile geometry shows good performance according to missile tailfin configuration at subsonic and supersonic speeds. The findings of study showed that multi-objective optimization problem can be solved by using modern computational fluid dynamics optimization technique. CFD solution method and the optimization procedure can be applied to design or optimize different geometry. In addition, the further study can be performed using asymmetric wing and tailfin configuration to investigate effect on missile aerodynamics.

\section{ACKNOWLEDGEMENTS}

This work is supported by the Scientific Research Projects Governing Unit of Gaziantep University (Project No. HUBF.17.04).

\section{REFERENCES}

Adeeb, E., A. Maqsood, A. Musthaq and C. H. Sohn (2016). Parametric Study and Optimization of Ceiling Fan Blades for Improved Aerodynamic Performance. Journal of Applied Fluid Mechanics 9(6), 2905-2916.
Aelaei, M., S. Karimian and F. Ommi (2019). Sensitivity Analysis and Optimization of Delta Wing Design Parameters using CFD-Based Response Surface Method. Journal of Applied Fluid Mechanics 12(6), 1885-1903.

Ageev, N. and A. Pavlenko (2016). Minimization of body of revolution aerodynamic drag at supersonic speeds. Aircraft Engineering and Aerospace Technology: An International Journal 88, 246-256.

Anderson, J. D. and J. Wendt (1995). Computational fluid dynamics. McGraw-Hill. New York, USA.

Anderson, M. B., J. E. Burkhalter and R. M. Jenkins (2000). Missile aerodynamic shape optimization using genetic algorithms. Journal of Spacecraft and Rockets 37, 663-669.

ANSYS Inc., (2009). Theory Guide, ANSYS Fluent 14.0

Box, G. E. and K. B. Wilson (1951). On the experimental attainment of optimum conditions. Journal of the royal statistical society: Series b (Methodological) 13(1), 1-38.

Deb, K. (1999). Multi-objective genetic algorithms: Problem difficulties and construction of test problems. Evolutionary computation 7(3), 205230.

Deck, S., P. Duveau, P. d'Espiney and P. Guillen (2002). Development and application of Spalart-Allmaras one equation turbulence model to three-dimensional supersonic complex configurations. Aerospace Science and Technology 6(3), 171-183

Dyer, J. D., R. J. Hartfield, G. V. Dozier and J. E. Burkhalter (2012). Aerospace design optimization using a steady state real-coded genetic algorithm. Applied Mathematics and Computation 218, 4710-4730

Gen, M. and R. Cheng (1997). Genetic Algorithms and Engineering Design. Wiley Interscience, MA, USA.

Goldberg, D. E. (1989). Genetic algorithms in search, optimization, and machine learning. Reading MA: Addison-Wesley.

Gülay, E., A. Akgül, J. Isaković and S. Mandić (2011). Computational Fluid Dynamics and Experimental Investigation of Wrap-AroundFins Missile Rolling Moment. Scientific Technical Review 61, 8-15.

Guy, Y., J. A. Morrow and T. E. McLaughlin (1999). The effects of canard shape on the aerodynamic characteristics of a generic missile configuration. AIAA Paper 99, 4256.

Haider, B. A., C. H. Sohn, Y. S. Won and Y. M. Koo (2017). Aerodynamically efficient rotor design for hovering agricultural unmanned helicopter. Journal of Applied Fluid 
A. Şumnu and İ. H. Güzelbey / JAFM, Vol. 14, No. 6, pp. 1795-1807, 2021.

Mechanics 10(5), 1461-1474.

Hao, W., W. Shaoping and M. M. Tomovic (2010). Modified sequential Kriging optimization for multidisciplinary complex product simulation. Chinese Journal of Aeronautics 23(5), 616-622.

Holland, J. H. (1975). Adaptation in Natural and Artificial Systems. The University of Michigan Press, Ann Arbor, Michigan.

Konak, A., D. W. Coit, and A. E. Smith (2006) Multi-objective optimization using genetic algorithms: A tutorial. Reliability Engineering \& System Safety 91(9): 992-1007.

Kumar, G. V. and D. A. Shah (2017). Aerodynamics of Flapping Wings for Vertical Takeoff. Journal of Applied Fluid Mechanics 10(6), 1689-1697.

Marler, R. T. and J. S. Arora (2004). Survey of multiobjective optimization methods for engineering. Structural and multidisciplinary optimization 26(6), 369-395.

Menter, F. R. (1994). Two-equation eddy-viscosity turbulence models for engineering applications. AIAA journal 32(8), 1598-1605.

Misaghian, B., M. R. Soltani, and A. Karimi (2007). Fin shape optimization to minimize aerodynamic heating using genetic algorithm, HEFAT.

Moghadam, Z. S. and A. Jahangirian (2017). Flow Control of Transonic Airfoils using Optimum Suction and Injection Parameters. Journal of Applied Fluid Mechanics 10(1), 103-115.

Munoz, P. J., J. Garcia, A. Crespo and F. Laspougeas (2015). Aerodynamic optimization of the nose shape of a train using the adjoint method. Journal of Applied Fluid Mechanics 8(3), 601612.

Nikbay, M., L. Öncü and A. Aysan (2009). Multidisciplinary code coupling for analysis and optimization of aeroelastic systems. Journal of Aircraft 46, 1938-1944.

Nobahari, H., Y. Nabavi and H. Pourtakdoust (2006). Aerodynamic Shape Optimization of Unguided Projectiles Using Ant Colony Optimization and Genetic Algorithm, In 25TH International Congress of the Aeronautical Sciences, Sharif University of Technology.

Oktay, E., N. Alemdaroğlu, E. Tarhan, P. Champigny and P. d'Espiney (2000). Unstructured Euler solutions for missile aerodynamics. Aerospace science and technology 4, 453-461.

Prabu, T., P. Viswanathan, N. G. Kannan, R. Rudramoorthy and A. Firthouse (2020). Optimization of Wrap Angle in the Mixed Flow Impeller for Reducing Flow Losses. Journal of
Applied Fluid Mechanics 13(6).

Rojas-Solórzano, L. R. (2017). Multi-Objective Optimization Techniques using ANSYS-CFXDesignXplorerTM. Case Study: Optimization of Static Mixer.

Runduo, C. and Z. Xiaobing (2018). Multi-objective optimization of the aerodynamic shape of a long-range guided rocket. Structural and Multidisciplinary Optimization 57, 1779-1792.

Ryan, K. (2011). Trajectory optimization and aerodynamic modeling of long range morphing projectiles. Doctoral dissertation, University of Maryland, College Park.

Sahu, J. and K. Heavey (2017). Computations of Supersonic Flow over a Complex Elliptical Missile Configuration. AIAA Paper, 5714

Silton, S. I. (2005). Navier-Stokes computations for a spinning projectile from subsonic to supersonic speeds. Journal of Spacecraft and Rockets 42, 223-231.

Smith, K. D. (2009). A Comparison of Two Inviscid Numerical Approaches to Modeling the Flow Field about a Supersonic Ballistic Object: The Method of Characteristics and a Finite Volume Euler Solution. Doctoral dissertation, Rensselaer Polytechnic Institute.

Sumnu, A., İ. H. Güzelbey and O. Öğücü (2020). Aerodynamic Shape Optimization of a Missile Using a Multiobjective Genetic Algorithm. International Journal of Aerospace Engineering 2020.

Supreeth, R., A. Arokkiaswamy, K. Anirudh, R. K. Pradyumna, P. K. Pramod and A. K. Sanarahamat (2020). Experimental and Numerical Investigation of the Influence of Leading Edge Tubercles on S823 Airfoil Behavior. Journal of Applied Fluid Mechanics 13(6), 1885-1899.

Tahani, M., M. Masdari and M. Kazemi (2017). Aerodynamic performance improvement of a canard control missile. Aircraft Engineering and Aerospace Technology 89, 871-878.

Tanil, C., B. Platin and G. Mahmutyazicioglu (2009, August). External configuration optimization of missiles in conceptual design. In AIAA Atmospheric Flight Mechanics Conference (p. 5719).

Vidanović, N. D., B. P. Rašuo, D. B. Damljanović, Đ. S. Vuković and D. S. Ćurčić (2014). Validation of the CFD code used for determination of aerodynamic characteristics of nonstandard AGARD-B calibration model. Thermal Science 18(4), 1223-1233.

Vidanović, N., B. Rašuo, G. Kastratović, S Maksimović, D. Curčić and M. Samardžić (2017). Aerodynamic-structural missile fin optimization. Aerospace Science and 
A. Şumnu and İ. H. Güzelbey / JAFM, Vol. 14, No. 6, pp. 1795-1807, 2021.

Technology 65, 26-45.

Yeong, S. P. and S. S. Dol (2016). Aerodynamic Optimization of Micro Aerial Vehicle. Journal of Applied Fluid Mechanics 9(5).

Yu, H. and Z. Yang (2020). A numerical simulation on the airfoil s833 equipped with flapping trailing edge fringes. Journal of Applied Fluid Mechanics 13(2), 571-582.

Zhang, W. D., Y. B. Wang, and Y. Liu (2013). Aerodynamic study of theater ballistic missile target. Aerospace Science and Technology 24, 221-225. 